\title{
A Stable Extrapolation Method for Multidimensional Degenerate Parabolic Problems*
}

\author{
By Ricardo H. Nochetto
}

\begin{abstract}
Degenerate parabolic problems in several space variables are approximated by combining a preliminary regularization procedure with a finite element extrapolation method. The proposed extrapolation acts on the so-called phase variable and leads to a linear problem which is shown to be stable. The ensuing linear algebraic system involves the same matrix for all time steps. Energy error estimates are also derived for the physical unknowns. An $O\left(h^{1 / 2}\right)$ rate of convergence is proved, provided the approximation parameters are suitably related. In case the linear systems are solved by an iterative algorithm, such as the conjugate gradient method, an $O\left(h^{3 / 2}\right)$ tolerance for the error reduction is shown to preserve the overall accuracy; the required computational effort is thus nearly optimal.
\end{abstract}

1. Introduction. So far, extrapolation methods have been mainly used for time discretization of mildly nonlinear parabolic PDE's [3], [4], [5], [6], [9]. The key idea consists of replacing at each time step a (mildly) nonlinear function of the solution by an expression involving only previous values of it. This leads to a linear elliptic PDE which can be further discretized in space. Efficient linear solvers can be eventually used to solve the resulting linear algebraic problem. The success of this technique is obviously based on having a quite regular solution in time. Such a regularity is unrealistic for degenerate parabolic equations because they give rise to rough solutions. There is indeed a lack of smoothness across the free boundaries which, in turn, are not known in advance [7], [10]. A first attempt to overcome this situation might be to resort to a preliminary regularization [10]. However, the strong regularity required for a classical extrapolation method to work cannot be expressed in terms of the regularization parameter, making the algorithm mathematically intractable.

The aim of this paper is to present a new extrapolation method for degenerate parabolic problems. A preliminary regularization is essential for the algorithm to work, but it requires minimal regularity properties to be stable. We shall be dealing with the following degenerate parabolic equation in the cylinder $Q=\Omega \times(0, T)$ :

$$
u_{t}-\Delta \theta=f(\theta), \quad u \in \gamma(\theta),
$$

where $\gamma$ denotes a maximal monotone graph with a singularity at the origin, $\Omega \subset \mathbf{R}^{d}$ $(d \geq 1)$ and $T<\infty$ is fixed; two-phase Stefan problems as well as porous medium equations are relevant examples. In addition, the graph $\gamma$ is assumed to have the

Received September 23, 1988.

1980 Mathematics Subject Classification (1985 Revision). Primary 65N15, 65N30, 35R35.

Key words and phrases. Mushy region, regularization, extrapolation, finite elements.

*This work was partially supported by NSF Grant DMS-8805218. 
form

$$
\gamma=\mu I+H
$$

where $\mu>0, H$ is also a maximal monotone graph and $I$ is the identity. For the simplest Stefan problem, $H$ can be chosen to be the Heaviside graph, thus representing the latent heat content. In this case, $H$ can be regarded as a phase variable, whereas $\theta$ is the temperature and $u$ the enthalpy (or energy density). We shall keep these names along the paper, even though their physical interpretation might be different.

The preliminary regularization procedure consists of replacing $H$ by a smooth function $H_{\varepsilon}$ whose maximal slope is bounded by $1 / \varepsilon ; \varepsilon>0$ is the regularization parameter. In order to introduce the extrapolation technique, it suffices to deal with a discrete-time algorithm; hereafter, $\tau:=T / N$ will indicate the time step. For $\Theta^{n}$ and $\chi^{n}$ being approximations to $\theta_{\varepsilon}(n \tau)$ and $H_{\varepsilon}\left(\theta_{\varepsilon}(n \tau)\right)(1 \leq n \leq N)$, respectively, the standard (nonlinear) scheme corresponds to enforcing the nonlinear constitutive relation $\chi_{\varepsilon}=H_{\varepsilon}\left(\theta_{\varepsilon}\right)$ also at the discrete level, namely,

$$
\chi^{n}=H_{\varepsilon}\left(\Theta^{n}\right), \quad 1 \leq n \leq N .
$$

This leads to a sequence of nonlinear elliptic problems [8], [13], [14], [19]. The present extrapolation procedure is motivated by the physical fact that whenever a mushy region occupies the entire domain $\Omega$, which turns out to be the most critical regularity situation, the function $H_{\varepsilon}\left(\theta_{\varepsilon}\right)$ coincides with $\varepsilon^{-1} \theta_{\varepsilon}$. We thus extrapolate $\chi^{n}$ as follows:

$$
\chi^{n}:=H_{\varepsilon}\left(\Theta^{n-1}\right)+\frac{1}{\varepsilon}\left(\Theta^{n}-\Theta^{n-1}\right), \quad 1 \leq n \leq N .
$$

This is a very crude extrapolation formula in that it utilizes time regularity for $\theta_{\varepsilon}$ rather than $\chi_{\varepsilon}$; note that $\chi_{\varepsilon}$ has very low regularity properties (uniformly in $\varepsilon$ ). In contrast to the standard scheme, the new elliptic problem is linear in the unknown $\Theta^{n}$, namely,

$$
\left(\mu+\frac{1}{\varepsilon}\right) \partial \Theta^{n}-\Delta \Theta^{n}=\partial\left[\frac{1}{\varepsilon} \Theta^{n-1}-H_{\varepsilon}\left(\Theta^{n-1}\right)\right]+f\left(\Theta^{n-1}\right),
$$

for $1 \leq n \leq N$, where $\partial \eta^{n}:=\tau^{-1}\left(\eta^{n}-\eta^{n-1}\right)$. We further discretize this problem in space by means of continuous piecewise linear finite elements for the variable $\Theta^{n}$ and piecewise constants for $\chi^{n}$. This is done in such a way that the right-hand side of (1.5) can be readily and exactly evaluated without using any quadrature formula. The matrix corresponding to the resulting algebraic problem is symmetric, positive definite and independent of $n$. Therefore, its Cholesky factorization may be computed only once at the very beginning, if a direct method is to be employed. In case the linear systems are solved by the conjugate gradient method, an approximate solution needs only be computed. Specifically, we shall show that an $O(\tau)$ tolerance for the error reduction is good enough to stop iterations and still preserve the overall accuracy; the required computational effort is thus nearly optimal. This result resembles that in [6], but its analysis differs from the original one. A different linearization technique has been recently examined in [12], [16], [17], [18]; see also [11], [15] for an account of the state-of-the-art on this subject. An incomplete iteration may be applied to this latter algorithm as well. 
The fully discrete scheme associated with (1.5) is shown to be stable in energy spaces provided the initial temperature $\theta_{0}$ satisfies $\theta_{0} \in H_{0}^{1}(\Omega)$; this ensures that $\theta_{t} \in L^{2}(Q)$. Since (1.4) can be written equivalently as

$$
\chi^{n}=H_{\varepsilon}\left(\Theta^{n-1}\right)+\frac{\tau}{\varepsilon} \partial \Theta^{n},
$$

we realize that a bit of time regularity for $\theta$ is expected to enhance stability. Energy error estimates are next derived for both physical unknowns $\theta$ and $u=\theta+\chi$. An $O\left(h^{1 / 2}\right)$ rate of convergence is proved under the mild constraints $\varepsilon=C_{1} h$, $\tau=C_{2} h^{3 / 2}$, where $C_{1}$ and $C_{2}$ are arbitrary positive constants; as usual, $h$ stands for the mesh size.

The paper is organized as follows. In Section 2 we state the precise assumptions and regularity properties, and we also introduce the fully discrete scheme. In Section 3 we deal with the discrete initial data. In Section 4 we demonstrate stability in the natural energy spaces, whereas we prove energy error estimates in Section 5 ; in both sections we assume that the fully discrete problem is solved exactly. We finally investigate in Section 6 the effect of an incomplete iteration process.

2. The Extrapolation Method. In this section we state hypotheses, set notation and formulate continuous and discrete problems.

2.1. Assumptions and Notation. Assume the following hypotheses on the data:

$\left(\mathbf{H}_{\Omega}\right) \quad \Omega \subset \mathbf{R}^{d}(d \geq 1)$ is a polyhedral and convex domain;

$\left(\mathbf{H}_{H}\right)$ the graph $H \subset \mathbf{R} \times \mathbf{R}$ has a singularity solely at the origin, is Lipschitz continuous elsewhere and satisfies: $0 \in H(0), \quad 0 \leq H^{\prime}(s) \leq H(s) / s$, a.e. on $\mathbf{R} \backslash\{0\}$ and $|H(s)| \leq a+b|s|$ for all $s \in \mathbf{R} \backslash\{0\} \quad(a, b \geq 0)$;

$\left(\mathbf{H}_{f}\right) \quad f: \mathbf{R} \rightarrow \mathbf{R}$ is a uniformly Lipschitz continuous function,i.e., $\mid f\left(s_{1}\right)-$ $f\left(s_{2}\right)|\leq F| s_{1}-s_{2} \mid, \quad$ for all $s_{1}, s_{2} \in \mathbf{R}$;

$\left(\mathbf{H}_{\theta_{0}}\right) \quad$ the initial temperature $\theta_{0}$ satisfies $\theta_{0} \in W_{0}^{1, \infty}(\Omega)$;

$\left(\mathbf{H}_{u_{0}}\right)$ denote by $\Omega^{+}, \Omega^{0}$ and $\Omega^{-}$the subdomains of $\Omega$ where $\theta_{0}>0, \theta_{0}=0$ and $\theta_{0}<0$, respectively, and assume that their (internal) boundaries $\Gamma^{+}=\partial \Omega^{+} \cap \Omega$ and $\Gamma^{-}=\partial \Omega^{-} \cap \Omega$ possess finite $(d-1)$-dimensional Hausdorff measure; in addition, the initial enthalpy $u_{0}$ satisfies $u_{0} \in \gamma\left(\theta_{0}\right)$ and $u_{0} \in C^{0,1 / 2}\left(\bar{\Omega}^{+}\right) \cap C^{0,1 / 2}\left(\bar{\Omega}^{0}\right) \cap$ $C^{0,1 / 2}\left(\bar{\Omega}^{-}\right)$.

Note that either set in $\left(\mathbf{H}_{u_{0}}\right)$ might be empty and that jump discontinuities across the initial interfaces $\Gamma^{+}$and $\Gamma^{-}$are allowed. Note also that the property $u_{0} \in C^{0,1 / 2}\left(\bar{\Omega}^{+}\right)\left(\right.$resp. $\left.C^{0,1 / 2}\left(\bar{\Omega}^{-}\right)\right)$results from $\left(\mathbf{H}_{\theta_{0}}\right)$ provided $H \in C^{0,1 / 2}([0, \infty))$ (resp. $\left.C^{0,1 / 2}((-\infty, 0])\right)$, as happens for the Stefan problem. Let $\left\{\mathfrak{S}_{h}\right\}_{h}$ be a family of partitions of $\Omega$ into triangular finite elements so that $\Omega=\bigcup_{S \in \mathfrak{S}_{h}} S$. We assume that:

$\left(\mathbf{H}_{\mathfrak{S}_{h}}\right)$ the family $\left\{\mathfrak{S}_{h}\right\}_{h}$ is regular and quasi-uniform [2, pp. 132, 140].

Quasi-uniformity is only used in defining the discrete initial temperature (see Section 3); it can be removed, thus allowing local refinements, provided $\theta_{0}$ satisfies a nondegeneracy property as in [13], [17]. We shall be dealing with finite element spaces $\mathbf{V}_{h}^{1}$ and $\mathbf{V}_{h}^{0}$ which satisfy

$$
\mathbf{V}_{h}^{1} \subset H_{0}^{1}(\Omega),\left.\quad \mathbf{V}_{h}^{1}\right|_{S}=P^{1}(S) ;\left.\quad \mathbf{V}_{h}^{0}\right|_{S}=P^{0}(S), \quad \forall S \in \mathfrak{S}_{h},
$$


where $P^{i}(S)$ indicates the space of polynomials of total degree not greater than $i$ restricted to $S$. We shall also need a pair of projection operators associated with these two spaces. The first one, called $P_{h}^{1}$, is a projection operator onto $\mathbf{V}_{h}^{1}$ defined by

$$
\left\langle\nabla P_{h}^{1} z, \nabla \phi\right\rangle=\langle\nabla z, \nabla \phi\rangle, \quad \forall z \in H_{0}^{1}(\Omega), \quad \phi \in \mathbf{V}_{h}^{1}
$$

Hereafter, $\langle\cdot, \cdot\rangle$ will indicate either the $L^{2}$-inner product or the pairing between $H_{0}^{1}(\Omega)$ and $H^{-1}(\Omega)$. The second operator is the $L^{2}$-projection onto $\mathbf{V}_{h}^{0}$, namely,

$$
\left\langle P_{h}^{0} z, \phi\right\rangle=\langle z, \phi\rangle, \quad \forall z \in L^{2}(\Omega), \quad \phi \in \mathbf{V}_{h}^{0} .
$$

In view of $\left(\mathbf{H}_{\Omega}\right),\left(\mathbf{H}_{\mathfrak{S}_{h}}\right)$ and (2.1) the following approximation properties hold [2]:

$$
\begin{array}{rlrl}
\left\|P_{h}^{1} z-z\right\|_{H^{s}(\Omega)} & \leq C h^{1-s}\|z\|_{H^{1}(\Omega)}, & & \forall 0 \leq s \leq 1, \quad z \in H_{0}^{1}(\Omega) \\
\left\|P_{h}^{0} z-z\right\|_{H^{-s}(\Omega)} \leq C h^{r+s}\|z\|_{H^{r}(\Omega)}, & & \forall 0 \leq s, r \leq 1, \quad z \in H^{r}(\Omega) .
\end{array}
$$

We conclude with some notation concerning the time discretization. Set $t^{n}:=$ $n \tau, I^{n}:=\left(t^{n-1}, t^{n}\right]$ and also $z^{n}:=z\left(\cdot, t^{n}\right)$ for all $1 \leq n \leq N$ and any continuous function in time defined in $Q$; recall that $\tau=T / N$ stands for the time step and $N$ is a positive integer. As usual, $C>0$ will indicate a constant independent of relevant parameters involved, but not necessarily the same at each occurrence.

2.2. Continuous Problems: Regularity. We now state the variational formulation of both (1.1) and its regularized counterpart along with their typical regularity properties.

Problem $(\mathbf{P})$. Find $\{u, \theta\}$ such that

$$
\begin{gathered}
u \in L^{\infty}\left(0, T ; L^{2}(\Omega)\right) \cap H^{1}\left(0, T ; H^{-1}(\Omega)\right), \quad \theta \in L^{2}\left(0, T ; H_{0}^{1}(\Omega)\right), \\
u(x, t) \in \gamma(\theta(x, t)), \quad \text { for a.e. }(x, t) \in Q, \\
u(\cdot, 0)=u_{0}
\end{gathered}
$$

and for a.e. $t \in(0, T)$ and all $\phi \in H_{0}^{1}(\Omega)$ the following equation holds,

$$
\left\langle\frac{\partial u}{\partial t}, \phi\right\rangle+\langle\nabla \theta, \nabla \phi\rangle=\langle f(\theta), \phi\rangle
$$

Existence and uniqueness of $(\mathbf{P})$ are well known as well as the following additional regularity result $[7],[10]$ :

$$
\text { if } \theta_{0} \in H_{0}^{1}(\Omega), \quad \text { then } \theta \in H^{1}\left(0, T ; L^{2}(\Omega)\right) \cap L^{\infty}\left(0, T ; H_{0}^{1}(\Omega)\right) .
$$

Let $\varepsilon>0$ denote the regularization parameter and $H_{\varepsilon}: \mathbf{R} \rightarrow \mathbf{R}$ be defined by

$$
H_{\varepsilon}(s):= \begin{cases}\min \left(H(s), \varepsilon^{-1} s\right), & s>0 \\ 0, & s=0 \\ \max \left(H(s), \varepsilon^{-1} s\right), & s<0 .\end{cases}
$$

Moreover, let $I_{\varepsilon}$ be the smallest interval so that $H_{\varepsilon}(s)=H(s)$ for all $s \in \mathbf{R} \backslash I_{\varepsilon}$ and set $\gamma_{\varepsilon}:=\mu I+H_{\varepsilon}, \beta_{\varepsilon}:=\gamma_{\varepsilon}^{-1}, \beta:=\gamma^{-1}$; note that $0 \in \bar{I}_{\varepsilon}$ and $\operatorname{meas}\left(I_{\varepsilon}\right) \leq$ $2 a \varepsilon /(1-b \varepsilon)$, thus $\left|\beta(s)-\beta_{\varepsilon}(s)\right| \leq C \varepsilon$ for all $s \in \mathbf{R}$. The regularized problem reads 
as follows:

Problem $\left(\mathbf{P}_{\varepsilon}\right)$. Find $\left\{u_{\varepsilon}, \theta_{\varepsilon}\right\}$ such that

$$
\begin{gathered}
u_{\varepsilon} \in L^{\infty}\left(0, T ; L^{2}(\Omega)\right) \cap H^{1}\left(0, T ; H^{-1}(\Omega)\right), \quad \theta_{\varepsilon} \in L^{2}\left(0, T ; H_{0}^{1}(\Omega)\right) \\
u_{\varepsilon}(x, t)=\gamma_{\varepsilon}\left(\theta_{\varepsilon}(x, t)\right), \quad \text { for a.e. }(x, t) \in Q \\
u_{\varepsilon}(\cdot, 0)=u_{0}
\end{gathered}
$$

and for all $t \in(0, T)$ and $\phi \in H_{0}^{1}(\Omega)$ we have

$$
\left\langle\frac{\partial u_{\varepsilon}}{\partial t}, \phi\right\rangle+\left\langle\nabla \theta_{\varepsilon}, \nabla \phi\right\rangle=\left\langle f\left(\theta_{\varepsilon}\right), \phi\right\rangle \text {. }
$$

The regularity required in (2.12) is valid uniformly in $\varepsilon$ but, in view of the choice of the initial enthalpy in (2.14), property (2.10) does no longer hold. Moreover, the bound $\left\|\frac{\partial u_{\varepsilon}}{\partial t}\right\|_{L^{2}(Q)} \leq C \varepsilon^{-1 / 2}$ used in previous works [8], [13], [14], [17], [19] fails to hold in the present situation.

2.3. Fully Discrete Scheme. Let $\Theta^{0} \in \mathbf{V}_{h}^{1}$ fulfil $\left\|\Theta^{0}\right\|_{H_{0}^{1}(\Omega)} \leq C$, where $C$ is independent of both $h$ and $\varepsilon$. In addition, set $\Theta^{-1}:=\Theta^{0}$ and define $\alpha_{\varepsilon}(s):=$ $\varepsilon^{-1} s-H_{\varepsilon}(s)$ for all $s \in \mathbf{R}$. The fully discrete extrapolation method is defined as follows:

Problem $\left(\mathbf{P}_{\varepsilon, h, \tau}\right)$. For any $1 \leq n \leq N$ find $\Theta^{n} \in \mathbf{V}_{h}^{1}$ such that

$$
\begin{aligned}
\left(\mu+\varepsilon^{-1}\right)\left\langle\partial P_{h}^{0} \Theta^{n}, \phi\right\rangle & +\left\langle\nabla \Theta^{n}, \nabla \phi\right\rangle \\
& =\left\langle\partial \alpha_{\varepsilon}\left(P_{h}^{0} \Theta^{n-1}\right), \phi\right\rangle+\left\langle f\left(P_{h}^{0} \Theta^{n-1}\right), \phi\right\rangle, \quad \forall \phi \in \mathbf{V}_{h}^{1} .
\end{aligned}
$$

The discrete phase variable and enthalpy are defined by

$$
\chi^{n}:=H_{\varepsilon}\left(P_{h}^{0} \Theta^{n-1}\right)+\varepsilon^{-1} P_{h}^{0}\left[\Theta^{n}-\Theta^{n-1}\right], \quad U^{n}:=\mu P_{h}^{0} \Theta^{n}+\chi^{n} .
$$

Since $P_{h}^{0} \Theta^{n-1}$ is constant on each finite element $S$ (it is actually the value of $\Theta^{n-1}$ at the barycenter of $S$ ), the right-hand side of $(2.16)$ can be readily and exactly evaluated. Consequently, (2.16) is a practical scheme in that it can be easily implemented on a computer. Let $\mathbf{M}$ and $\mathbf{K}$ denote the matrices

$$
\mathbf{M}:=\left(\left\langle\phi_{i}, \psi_{j}\right\rangle\right)_{i, j=1}^{J}, \quad \mathbf{K}:=\left(\left\langle\nabla \phi_{i}, \nabla \phi_{j}\right\rangle\right)_{i, j=1}^{J},
$$

where $\phi_{i}$ is the $i$ th element of the canonical basis of $\mathbf{V}_{h}^{1}$ and $\psi_{j}$ is the characteristic function of $\operatorname{supp} \phi_{j}$ multiplied by $(d+1)^{-1}$. Equation (2.16) can then be written equivalently in matrix form as follows:

$$
\mathbf{A} \Theta^{n}:=\left[\mathbf{M}+\frac{\varepsilon \tau}{1+\mu \varepsilon} \mathbf{K}\right] \Theta^{n}=R^{n-1},
$$

where $R^{n-1}$ depends only on $\Theta^{n-1}$. We see that this problem is linear and that the corresponding matrix is symmetric, positive definite and also independent of $n$. This yields unique solvability of (2.16). When the bandwidth of the system (2.18) is reasonably small, a Cholesky factorization is recommended for its effective resolution [1]. Such a factorization is to be performed only once at the beginning. Otherwise, when dealing with large bandwidths, such as those arising from adaptive algorithms, the (preconditioned) conjugate gradient method is preferred for solving (2.18) [1], [16], [18]. The condition number $k(\mathbf{A})$ of $\mathbf{A}$, and thus that of an eventual preconditioned matrix, is bounded uniformly in $h$ because

$$
\Lambda h^{d} \leq \frac{\mathbf{x} \cdot \mathbf{A x}}{\|\mathbf{x}\|_{2}^{2}} \leq \Lambda^{-1}\left(1+\varepsilon \tau h^{-2}\right) h^{d}, \quad \forall \mathbf{x} \in \mathbf{R}^{J}
$$


and $\varepsilon \tau=o\left(h^{2}\right)$ (see Section 5). Here, $\Lambda>0$ indicates a constant independent of $h, \varepsilon$ and $\tau$. Consequently, the error reduction produced by the conjugate gradient method is given by

$$
\rho_{k}:=2\left(\frac{k(\mathbf{A})^{1 / 2}-1}{k(\mathbf{A})^{1 / 2}+1}\right)^{k}=2 Q^{k},
$$

where $k \in \mathbf{N}$ stands for the number of iterations [1] and $Q$ is bounded uniformly in $h$. It will be shown in Section 6 that an $O(\tau)$ tolerance for $\rho_{k}$ can be used safely as a stopping criterion; hence, the lower bound

$$
k \geq k_{0}:=\log \tau / \log Q
$$

is almost uniformly bounded for computational purposes. As a result, the associated incomplete iteration process discussed in Section 6 involves a nearly optimal computational labor.

3. Discrete Initial Data. Our present task is that of selecting the initial temperature $\Theta^{0} \in \mathbf{V}_{h}^{1}$. We wish the following two properties to hold:

$$
\left\|\Theta^{0}\right\|_{H_{0}^{1}(\Omega)} \leq C, \quad\left\|U^{0}-u_{0}\right\|_{H^{-1}(\Omega)} \leq C h^{1 / 2},
$$

where $U^{0}$, the initial enthalpy, is defined by $U^{0}:=\mu P_{h}^{0} \Theta^{0}+H_{\varepsilon}\left(P_{h}^{0} \Theta^{0}\right)$. The obvious choices $\Theta^{0}=P_{h}^{1} \theta_{0}$ and $\Theta^{0}=I_{h} \theta_{0}$ ( $I_{h}$ interpolation operator) fail to work in this context because the corresponding $U^{0}$ does not satisfy the error bound in (3.1), unless an initial nondegeneracy property is verified [13], [17]. This condition prevents Problem $(\mathbf{P})$ from having an initial mushy region. Since their presence is the major motivation for our extrapolation method, we would like to allow mushy regions from the very beginning, as stated in $\left(\mathbf{H}_{u_{0}}\right)$. Moreover, $\left(\mathbf{H}_{u_{0}}\right)$ together with $\left(\mathbf{H}_{\theta_{0}}\right)$ implies that $u_{0}$, and thus $\beta_{\varepsilon}\left(u_{0}\right)$, is continuous everywhere but on the initial interfaces $\Gamma^{+}$and $\Gamma^{-}$, where jump discontinuities might occur. We then define $\Theta^{0} \in \mathbf{V}_{h}^{1}$ at each node $x_{i}$ to be

$$
\Theta_{i}^{0}:=\beta_{\varepsilon}\left(\limsup _{x \rightarrow x_{i}} u_{0}(x)\right) ;
$$

note that $\Theta_{i}^{0}=\beta_{\varepsilon}\left(u_{0}\left(x_{i}\right)\right)$ for all $x_{i} \notin \Gamma^{+} \cup \Gamma^{-}$. Hence, $\Theta^{0}$ is easy to evaluate in practice.

LEMMA 3.1. The initial data $\Theta^{0}$ and $U^{0}$ satisfy (3.1) provided $\varepsilon$ and $h$ are subject to the mild constraint $\varepsilon^{2} \leq C^{*} h$, where $C^{*}>0$ is an arbitrary constant.

Proof. Let $S \in \mathfrak{S}_{h}$ be given and let $x_{i}$ and $x_{j}$ denote two of its vertices verifying $\Theta^{0}\left(x_{i}\right) \geq \Theta^{0}\left(x_{j}\right)$. In order to demonstrate the a priori estimate in (3.1), we distinguish three cases depending upon where $S$ is located. Let $\Omega_{\varepsilon}^{0}$ be defined by

$$
\Omega_{\varepsilon}^{0}:=\left\{x \in \Omega: u_{0}(x) \in \gamma_{\varepsilon}\left(\bar{I}_{\varepsilon}\right)\right\} .
$$

Assume first that $S \subset \Omega_{\varepsilon}^{0} \backslash\left(\Gamma^{+} \cup \Gamma^{-}\right)$. Since $\beta_{\varepsilon}(s)=\varepsilon s /(1+\varepsilon \mu)$ for all $s \in \gamma_{\varepsilon}\left(\bar{I}_{\varepsilon}\right)$, we can write

$$
\Theta^{0}\left(x_{i}\right)-\Theta^{0}\left(x_{j}\right)=\beta_{\varepsilon}\left(u_{0}\left(x_{i}\right)\right)-\beta_{\varepsilon}\left(u_{0}\left(x_{j}\right)\right)=\frac{\varepsilon}{1+\varepsilon \mu}\left[u_{0}\left(x_{i}\right)-u_{0}\left(x_{j}\right)\right],
$$

whence $\left\|\nabla \Theta^{0}\right\|_{L^{\infty}(S)} \leq C \varepsilon h^{-1 / 2}$. Here we have used $\left(\mathbf{H}_{\mathfrak{S}_{h}}\right)$ as well as $\left(\mathbf{H}_{u_{0}}\right)$. Suppose now that $S \cap\left(\Gamma^{+} \cup \Gamma^{-}\right) \neq \varnothing$, and denote by $F$ the union of all finite elements 
having this property. Since both $\Gamma^{+}$and $\Gamma^{-}$possess finite $(d-1)$-dimensional Hausdorff measure, we infer that meas $(F) \leq C h$, where meas stands for the Lebesgue measure. Hence, in view of the property $\beta\left(\lim \sup _{x \rightarrow x_{0}} u_{0}(x)\right)=\theta_{0}\left(x_{0}\right)$ for all $x_{0} \in \Omega$, we arrive at

$$
\begin{aligned}
\Theta^{0}\left(x_{i}\right)-\Theta^{0}\left(x_{j}\right)= & \beta_{\varepsilon}\left(\limsup _{x \rightarrow x_{i}} u_{0}(x)\right)-\beta_{\varepsilon}\left(\limsup _{x \rightarrow x_{j}} u_{0}(x)\right) \\
= & \beta_{\varepsilon}\left(\limsup _{x \rightarrow x_{i}} u_{0}(x)\right)-\beta\left(\limsup _{x \rightarrow x_{i}} u_{0}(x)\right) \\
& +\theta_{0}\left(x_{i}\right)-\theta_{0}\left(x_{j}\right) \\
& +\beta\left(\limsup _{x \rightarrow x_{j}} u_{0}(x)\right)-\beta_{\varepsilon}\left(\limsup _{x \rightarrow x_{j}} u_{0}(x)\right) \\
\leq & C(\varepsilon+h),
\end{aligned}
$$

which, coupled with an inverse inequality, results in

$$
\left\|\nabla \Theta^{0}\right\|_{L^{2}(F)} \leq C h^{-1}(\varepsilon+h) \operatorname{meas}(F)^{1 / 2} \leq C\left(\varepsilon h^{-1 / 2}+h^{1 / 2}\right) .
$$

We next consider the case $S \subset \Omega^{+}$and $x_{i} \in \Omega^{+} \backslash \Omega_{\varepsilon}^{0}$; one can argue with $\Omega^{-}$in a quite similar fashion. It follows that

$$
\begin{gathered}
\Theta^{0}\left(x_{i}\right)=\beta_{\varepsilon}\left(u_{0}\left(x_{i}\right)\right)=\theta_{0}\left(x_{i}\right), \\
\Theta^{0}\left(x_{j}\right)=\beta_{\varepsilon}\left(u_{0}\left(x_{j}\right)\right) \geq \beta\left(u_{0}\left(x_{j}\right)\right)=\theta_{0}\left(x_{j}\right),
\end{gathered}
$$

whence, by $\left(\mathbf{H}_{\theta_{0}}\right)$,

$$
\Theta^{0}\left(x_{i}\right)-\Theta^{0}\left(x_{j}\right) \leq \theta_{0}\left(x_{i}\right)-\theta_{0}\left(x_{j}\right) \leq C h .
$$

This yields $\left\|\nabla \Theta^{0}\right\|_{L^{\infty}(S)} \leq C$ and completes the proof of the first estimate in (3.1). The remaining one will be derived in $L^{2}(\Omega)$ rather than $H^{-1}(\Omega)$. Let $S$ satisfy $S \cap\left(\Gamma^{+} \cup \Gamma^{-}\right)=\varnothing$. Since $u_{0}$ is Hölder continuous on $S$, there exists $y \in S$ such that $P_{h}^{0} \Theta^{0}(x)=\beta_{\varepsilon}\left(u_{0}(y)\right)$ for all $x \in S$. Then

$$
U^{0}(x)-u_{0}(x)=\gamma_{\varepsilon}\left(\beta_{\varepsilon}\left(u_{0}(y)\right)\right)-u_{0}(x)=u_{0}(y)-u_{0}(x), \quad \forall x \in S,
$$

which, in turn, leads to $\left\|U^{0}-u_{0}\right\|_{L^{\infty}(S)} \leq C h^{1 / 2}$. Finally, the fact that $\operatorname{meas}(F) \leq$ $C h$ implies $\left\|U^{0}-u_{0}\right\|_{L^{2}(F)} \leq C h^{1 / 2}$ and concludes the proof of the lemma.

4. Stability. The aim of this section is to show that the fully discrete scheme (2.16) satisfies a discrete version of (2.10).

LEMMA 4.1. There exists a constant $C>0$ independent of $h, \varepsilon$ and $\tau$ such that

$$
\sum_{n=1}^{N} \tau\left\|\partial P_{h}^{0} \Theta^{n}\right\|_{L^{2}(\Omega)}^{2}+\max _{1 \leq n \leq N}\left\|\nabla \Theta^{n}\right\|_{L^{2}(\Omega)}^{2} \leq C\left(1+\left\|\nabla \Theta^{0}\right\|_{L^{2}(\Omega)}^{2}\right)
$$


Proof. Take $\phi:=\tau \partial \Theta^{n} \in \mathbf{V}_{h}^{1}$ as a test function in (2.16), add on $n$ from 1 to $m \leq N$ and use definition (2.3) of $P_{h}^{0}$ to rewrite the resulting expression as follows:

$$
\begin{aligned}
\left(\mu+\varepsilon^{-1}\right) & \sum_{n=1}^{m} \tau\left\langle\partial P_{h}^{0} \Theta^{n}, \partial P_{h}^{0} \Theta^{n}\right\rangle+\sum_{n=1}^{m}\left\langle\nabla \Theta^{n}, \nabla\left[\Theta^{n}-\Theta^{n-1}\right]\right\rangle \\
& =: \mathrm{I}+\mathrm{II} \\
& =\sum_{n=1}^{m} \tau\left\langle\partial \alpha_{\varepsilon}\left(P_{h}^{0} \Theta^{n-1}\right), \partial P_{h}^{0} \Theta^{n}\right\rangle+\sum_{n=1}^{m} \tau\left\langle f\left(P_{h}^{0} \Theta^{n-1}\right), \partial P_{h}^{0} \Theta^{n}\right\rangle \\
& =: \text { III }+ \text { IV. }
\end{aligned}
$$

We now proceed to estimate the last three terms separately. Making use of the elementary identity

$$
2 a \cdot[a-b]=|a|^{2}-|b|^{2}+|a-b|^{2} \quad \text { for } a, b \in \mathbf{R}^{d},
$$

we easily get

$$
\begin{aligned}
\mathrm{II} & =\frac{1}{2} \sum_{n=1}^{m}\left(\left\|\nabla \Theta^{n}\right\|_{L^{2}(\Omega)}^{2}-\left\|\nabla \Theta^{n-1}\right\|_{L^{2}(\Omega)}^{2}+\left\|\nabla\left[\Theta^{n}-\Theta^{n-1}\right]\right\|_{L^{2}(\Omega)}^{2}\right) \\
& \geq \frac{1}{2}\left\|\nabla \Theta^{m}\right\|_{L^{2}(\Omega)}^{2}-\frac{1}{2}\left\|\nabla \Theta^{0}\right\|_{L^{2}(\Omega)}^{2} .
\end{aligned}
$$

In evaluating the contribution of III we observe that the function $\alpha_{\varepsilon}$ satisfies $0 \leq$ $\alpha_{\varepsilon}^{\prime}(s) \leq \varepsilon^{-1}$ a.e. $s \in \mathbf{R}$. Hence,

$$
\mathrm{III} \leq \varepsilon^{-1} \sum_{n=1}^{m} \tau\left\|\partial P_{h}^{0} \Theta^{n-1}\right\|_{L^{2}(\Omega)}\left\|\partial P_{h}^{0} \Theta^{n}\right\|_{L^{2}(\Omega)} \leq \varepsilon^{-1} \sum_{n=1}^{m} \tau\left\|\partial P_{h}^{0} \Theta^{n}\right\|_{L^{2}(\Omega)}^{2},
$$

because $\Theta^{-1}=\Theta^{0}$; this term can be readily absorbed into the left-hand side of (4.2). Assumption $\left(\mathbf{H}_{f}\right)$, together with

$$
P_{h}^{0} \Theta^{n-1}=P_{h}^{0} \Theta^{0}+\sum_{i=1}^{n-1} \tau \partial P_{h}^{0} \Theta^{i}, \quad 2 \leq n \leq N
$$

results in

$$
\begin{aligned}
\mathrm{IV} & \leq C \sum_{n=1}^{m} \tau\left\|\partial P_{h}^{0} \Theta^{n}\right\|_{L^{2}(\Omega)}\left(1+\left\|P_{h}^{0} \Theta^{n-1}\right\|_{L^{2}(\Omega)}\right) \\
& \leq \sum_{n=1}^{m} \tau\left[\frac{\mu}{2}\left\|\partial P_{h}^{0} \Theta^{n}\right\|_{L^{2}(\Omega)}^{2}+C\left(\sum_{i=1}^{n} \tau\left\|\partial P_{h}^{0} \Theta^{i}\right\|_{L^{2}(\Omega)}\right)^{2}+C\left(1+\left\|\Theta^{0}\right\|_{L^{2}(\Omega)}^{2}\right)\right] \\
& \leq \frac{\mu}{2} \sum_{n=1}^{m} \tau\left\|\partial P_{h}^{0} \Theta^{n}\right\|_{L^{2}(\Omega)}^{2}+C \sum_{n=1}^{m} \sum_{i=1}^{n} \tau^{2}\left\|\partial P_{h}^{0} \Theta^{i}\right\|_{L^{2}(\Omega)}^{2}+C\left(1+\left\|\nabla \Theta^{0}\right\|_{L^{2}(\Omega)}^{2}\right) .
\end{aligned}
$$

Collecting the above estimates and inserting them in (4.2), we easily obtain

$$
\begin{aligned}
\mu \sum_{n=1}^{m} \tau\left\|\partial P_{h}^{0} \Theta^{n}\right\|_{L^{2}(\Omega)}^{2} & +\left\|\nabla \Theta^{m}\right\|_{L^{2}(\Omega)}^{2} \\
& \leq C\left(1+\left\|\nabla \Theta^{0}\right\|_{L^{2}(\Omega)}^{2}\right)+C \sum_{n=1}^{m} \sum_{i=1}^{n} \tau^{2}\left\|\partial P_{h}^{0} \Theta^{i}\right\|_{L^{2}(\Omega)}^{2} .
\end{aligned}
$$


The discrete Gronwall lemma finally yields the assertion.

Remark 4.1. Note that term III above can also be bounded by

$$
\text { III } \leq \varepsilon^{-1} \sum_{n=1}^{m-1} \tau\left\|\partial P_{h}^{0} \Theta^{n}\right\|_{L^{2}(\Omega)}^{2}+\tau(2 \varepsilon)^{-1}\left\|\partial P_{h}^{0} \Theta^{m}\right\|_{L^{2}(\Omega)}^{2} .
$$

Consequently, arguing as before, we find the additional estimate

$$
\max _{1 \leq n \leq N}\left\|P_{h}^{0}\left[\Theta^{n}-\Theta^{n-1}\right]\right\|_{L^{2}(\Omega)} \leq C(\varepsilon \tau)^{1 / 2}\left(1+\left\|\nabla \Theta^{0}\right\|_{L^{2}(\Omega)}\right) .
$$

The next a priori estimate resembles that in (2.6) for the continuous enthalpy $u$ because $\tau$ and $\varepsilon$ will be related in such a way that $\tau=o(\varepsilon)$.

LEMMA 4.2. There exists a constant $C>0$ independent of $h, \varepsilon$ and $\tau$ such that

$$
\max _{1 \leq n \leq N}\left\|U^{n}\right\|_{L^{2}(\Omega)} \leq C\left(1+\tau^{1 / 2} \varepsilon^{-1 / 2}\right)\left(1+\left\|\nabla \Theta^{0}\right\|_{L^{2}(\Omega)}\right) .
$$

Proof. According to (2.17) we have

$$
U^{n}=\mu P_{h}^{0} \Theta^{n}+H_{\varepsilon}\left(P_{h}^{0} \Theta^{n-1}\right)+\varepsilon^{-1} P_{h}^{0}\left[\Theta^{n}-\Theta^{n-1}\right] .
$$

Making use of (4.4) coupled with (4.1), we easily conclude that

$$
\max _{1 \leq n \leq N}\left\|P_{h}^{0} \Theta^{n}\right\|_{L^{2}(\Omega)} \leq C .
$$

This and the fact that $H$ grows at most linearly at infinity, as stated in $\left(\mathbf{H}_{H}\right)$, yields $\max _{1 \leq n \leq N}\left\|H_{\varepsilon}\left(P_{h}^{0} \Theta^{n-1}\right)\right\|_{L^{2}(\Omega)} \leq C$. Finally, in dealing with the rightmost term in (4.7) we resort to (4.5). These estimates clearly give the desired result.

5. Error Analysis. In this section we shall demonstrate various energy error estimates for the physical variables $\theta$ and $u$. Before we get started, let us introduce some further notation:

$$
\begin{aligned}
e_{\theta}^{\varepsilon}:=\theta-\theta_{\varepsilon}, \quad e_{u}^{\varepsilon} & :=u-u_{\varepsilon} ; \quad e_{u}^{0}:=u_{0}-U^{0} \\
e_{\theta}^{h}(t):=\theta_{\varepsilon}(t)-\Theta^{n}, \quad e_{u}^{h}(t) & :=u_{\varepsilon}(t)-U^{n}, \quad \forall t \in I^{n}, \quad 1 \leq n \leq N .
\end{aligned}
$$

We first recall a well-known result regarding the effect of the regularization procedure [8], [13], [14], [17], [19].

THEOREM 5.1. There exists a constant $C>0$ independent of $\varepsilon$ such that

$$
\left\|e_{\theta}^{\varepsilon}\right\|_{L^{2}(Q)}+\left\|\int_{0}^{t} e_{\theta}^{\varepsilon}\right\|_{L^{\infty}\left(0, T ; H_{0}^{1}(\Omega)\right)}+\left\|e_{u}^{\varepsilon}\right\|_{L^{\infty}\left(0, T ; H^{-1}(\Omega)\right)} \leq C \varepsilon^{1 / 2} .
$$

The next two results are independent of the particular choice of $\Theta^{0}$ made in Section 3 ; hence, they are valid for regular meshes that may have local refinements. Our main concern now is to prove an error estimate for the fully discrete temperature.

THEOREM 5.2. There exists a constant $C>0$ independent of $h, \varepsilon$ and $\tau$ such that

$$
\left\|e_{\theta}^{h}\right\|_{L^{2}(Q)}+\left\|\int_{0}^{t} e_{\theta}^{h}\right\|_{L^{\infty}\left(0, T ; H_{0}^{1}(\Omega)\right)} \leq C\left(h \varepsilon^{-1 / 2}+\tau \varepsilon^{-1}+\tau^{1 / 2}+\left\|e_{u}^{0}\right\|_{H^{-1}(\Omega)}\right) .
$$


Proof. Integrate Eq. (2.15) on $I^{i}$ for $1 \leq i \leq N$ to arrive at

$$
\left\langle\partial u_{\varepsilon}^{i}, \phi\right\rangle+\tau^{-1}\left\langle\nabla \int_{I^{i}} \theta_{\varepsilon}, \nabla \phi\right\rangle=\tau^{-1}\left\langle\int_{I^{i}} f\left(\theta_{\varepsilon}\right), \phi\right\rangle, \quad \forall \phi \in H_{0}^{1}(\Omega)
$$

On the other hand, Eq. (2.16) can also be written as follows:

$$
\begin{aligned}
\left\langle\partial \gamma_{\varepsilon}\left(P_{h}^{0} \Theta^{i}\right), \phi\right\rangle+ & \left\langle\nabla \Theta^{i}, \nabla \phi\right\rangle \\
= & \left\langle\partial\left[\alpha_{\varepsilon}\left(P_{h}^{0} \Theta^{i-1}\right)-\alpha_{\varepsilon}\left(P_{h}^{0} \Theta^{i}\right)\right], \phi\right\rangle \\
& +\left\langle f\left(P_{h}^{0} \Theta^{i-1}\right), \phi\right\rangle, \quad \forall \phi \in \mathbf{V}_{h}^{1}, \quad 1 \leq i \leq N .
\end{aligned}
$$

Subtracting these two expressions, adding over $i$ from 1 to $n \leq N$ and recalling (2.13) as well as $\Theta^{-1}=\Theta^{0}$ and $U^{0}:=\mu P_{h}^{0} \Theta^{0}+H_{\varepsilon}\left(P_{h}^{0} \Theta^{0}\right)$, we easily derive the following error equation for $1 \leq n \leq N$ :

$$
\begin{aligned}
\left\langle\gamma_{\varepsilon}\left(\theta_{\varepsilon}^{n}\right)-\right. & \left.\gamma_{\varepsilon}\left(P_{h}^{0} \Theta^{n}\right), \phi\right\rangle+\left\langle\sum_{i=1}^{n} \nabla \int_{I^{i}} e_{\theta}^{h}(t) d t, \nabla \phi\right\rangle \\
= & \left\langle e_{u}^{0}, \phi\right\rangle \\
& +\left\langle\alpha_{\varepsilon}\left(P_{h}^{0} \Theta^{n}\right)-\alpha_{\varepsilon}\left(P_{h}^{0} \Theta^{n-1}\right), \phi\right\rangle \\
& +\left\langle\sum_{i=1}^{n} \int_{I^{i}}\left[f\left(\theta_{\varepsilon}(t)\right)-f\left(P_{h}^{0} \Theta^{i-1}\right)\right] d t, \phi\right\rangle, \quad \forall \phi \in \mathbf{V}_{h}^{1} .
\end{aligned}
$$

To proceed further, we make use of a technique introduced in [13]. Namely, take $\phi:=\int_{I^{n}} P_{h}^{1} e_{\theta}^{h}(t) d t \in \mathbf{V}_{h}^{1}$ as a test function in (5.3) and next add these equations from $n=1$ to $n=m \leq N$. The resulting expression reads as follows:

$$
\begin{aligned}
\sum_{n=1}^{m} \int_{I^{n}}\left\langle\gamma_{\varepsilon}\left(\theta_{\varepsilon}^{n}\right)-\gamma_{\varepsilon}\left(P_{h}^{0} \Theta^{n}\right), P_{h}^{1} e_{\theta}^{h}(t)\right\rangle d t & \\
& +\sum_{n=1}^{m}\left\langle\sum_{i=1}^{n} \nabla \int_{I^{i}} e_{\theta}^{h}(s) d s, \nabla \int_{I^{n}} P_{h}^{1} e_{\theta}^{h}(t) d t\right\rangle \\
= & \mathrm{I}+\mathrm{II} \\
= & \left\langle e_{u}^{0}, \sum_{n=1}^{m} \int_{I^{n}} P_{h}^{1} e_{\theta}^{h}(t) d t\right\rangle \\
& +\sum_{n=1}^{m} \tau\left\langle\partial \alpha_{\varepsilon}\left(P_{h}^{0} \Theta^{n}\right), \int_{I^{n}} P_{h}^{1} e_{\theta}^{h}(t) d t\right\rangle \\
& +\sum_{n=1}^{m}\left\langle\sum_{i=1}^{n} \int_{I^{i}}\left[f\left(\theta_{\varepsilon}(s)\right)-f\left(P_{h}^{0} \Theta^{i-1}\right)\right] d s, \int_{I^{n}} P_{h}^{1} e_{\theta}^{h}(t) d t\right\rangle \\
= & \mathrm{III}+\mathrm{IV}+\mathrm{V}, \quad \forall 1 \leq m \leq N .
\end{aligned}
$$


The rest of this proof consists of simply estimating these five terms. To begin with, we rewrite term $I$ in a more convenient form, namely,

$$
\begin{aligned}
\mathrm{I}= & \sum_{n=1}^{m} \int_{I^{n}}\left\langle\gamma_{\varepsilon}\left(\theta_{\varepsilon}(t)\right)-\gamma_{\varepsilon}\left(P_{h}^{0} \Theta^{n}\right), \theta_{\varepsilon}(t)-P_{h}^{0} \Theta^{n}\right\rangle d t \\
& +\sum_{n=1}^{m} \int_{I^{n}}\left\langle u_{\varepsilon}^{n}-u_{\varepsilon}(t), P_{h}^{1} e_{\theta}^{h}(t)\right\rangle d t \\
& +\sum_{n=1}^{m} \int_{I^{n}}\left\langle\gamma_{\varepsilon}\left(\theta_{\varepsilon}(t)\right)-\gamma_{\varepsilon}\left(P_{h}^{0} \Theta^{n}\right),\left[P_{h}^{1}-I\right] \theta_{\varepsilon}(t)\right\rangle d t \\
& +\sum_{n=1}^{m} \int_{I^{n}}\left\langle\gamma_{\varepsilon}\left(\theta_{\varepsilon}(t)\right)-\gamma_{\varepsilon}\left(P_{h}^{0} \Theta^{n}\right),\left[P_{h}^{0}-I\right] \Theta^{n}\right\rangle d t \\
= & : \mathrm{I}_{1}+\mathrm{I}_{2}+\mathrm{I}_{3}+\mathrm{I}_{4} .
\end{aligned}
$$

By virtue of the definition of $\gamma_{\varepsilon}$, as well as (2.5), (4.1) and the property $\beta_{\varepsilon}^{\prime} \geq$ $\varepsilon /(1+\varepsilon \mu) \geq \varepsilon / 2$, we can write

$$
\begin{aligned}
\mathrm{I}_{1} \geq & \frac{\mu}{2} \sum_{n=1}^{m} \int_{I^{n}}\left\|\theta_{\varepsilon}(t)-P_{h}^{0} \Theta^{n}\right\|_{L^{2}(\Omega)}^{2} d t \\
& +\frac{\varepsilon}{4} \sum_{n=1}^{m} \int_{I^{n}}\left\|\gamma_{\varepsilon}\left(\theta_{\varepsilon}(t)\right)-\gamma_{\varepsilon}\left(P_{h}^{0} \Theta^{n}\right)\right\|_{L^{2}(\Omega)}^{2} d t \\
\geq & \frac{\mu}{2}\left\|e_{\theta}^{h}\right\|_{L^{2}\left(0, t^{m} ; L^{2}(\Omega)\right)}^{2}+\frac{\varepsilon}{4}\left\|e_{\gamma_{\varepsilon}(\theta)}\right\|_{L^{2}\left(0, t^{m} ; L^{2}(\Omega)\right)}^{2}-C h^{2}
\end{aligned}
$$

where $e_{\gamma_{\varepsilon}(\theta)}:=\gamma_{\varepsilon}\left(\theta_{\varepsilon}\right)-\gamma_{\varepsilon}\left(P_{h}^{0} \Theta^{n}\right)$. The contribution due to $\mathrm{I}_{2}$ is easily bounded in view of $(2.4),(2.12)$ and (4.1). In fact, we have

$$
\mathrm{I}_{2} \leq \sum_{n=1}^{m} \int_{I^{n}}\left\|\int_{t}^{t^{n}} \frac{\partial u_{\varepsilon}}{\partial s}(s) d s\right\|_{H^{-1}(\Omega)}\left(\left\|\theta_{\varepsilon}(t)\right\|_{H^{1}(\Omega)}+\left\|\Theta^{n}\right\|_{H^{1}(\Omega)}\right) d t \leq C \tau .
$$

The last two terms are similar and, thus, can be analyzed in the same manner. By (2.4) and (2.12) (resp. (2.5) and (4.1)), we obtain

$$
\mathrm{I}_{3}\left(\text { resp. } \mathrm{I}_{4}\right) \leq \frac{\varepsilon}{8}\left\|e_{\gamma_{\varepsilon}(\theta)}\right\|_{L^{3}\left(0, t^{m} ; L^{2}(\Omega)\right)}^{2}+C \frac{h^{2}}{\varepsilon},
$$

where $C>0$ depends on $\left\|\theta_{\varepsilon}\right\|_{L^{2}\left(0, T ; H_{0}^{1}(\Omega)\right)}\left(\right.$ resp. $\left.\sum_{n=1}^{N} \tau\left\|\Theta^{n}\right\|_{H^{1}(\Omega)}^{2}\right)$. The above estimates lead to the following bound for I:

$$
\mathrm{I} \geq \frac{\mu}{2}\left\|e_{\theta}^{h}\right\|_{L^{2}\left(0, t^{m} ; L^{2}(\Omega)\right)}^{2}-C\left(\frac{h^{2}}{\varepsilon}+\tau\right) .
$$

The next term in (5.4) provides the $H^{1}$-estimate. In order to prove it, we first need the following by-product of (4.3):

$$
2 \sum_{n=1}^{m} a_{n} \cdot\left(\sum_{i=1}^{n} a_{i}\right)=\left(\sum_{n=1}^{m} a_{n}\right)^{2}+\sum_{n=1}^{m} a_{n}^{2}, \quad \forall a_{n} \in \mathbf{R}^{d}, \quad 1 \leq m \leq N
$$

Next, take $a_{n}=\nabla \int_{I^{n}} P_{h}^{1} e_{\theta}^{h}(t) d t$ and use this identity to deduce

$$
\mathrm{II} \geq \frac{1}{2}\left\|\sum_{n=1}^{m} \int_{I^{n}} \nabla P_{h}^{1} e_{\theta}^{h}(t) d t\right\|_{L^{2}(\Omega)}^{2}=\frac{1}{2}\left\|\int_{0}^{t^{m}} \nabla P_{h}^{1} e_{\theta}^{h}(t) d t\right\|_{L^{2}(\Omega)}^{2} .
$$


For term III we use the duality between $H_{0}^{1}(\Omega)$ and $H^{-1}(\Omega)$ to arrive at

$$
\begin{aligned}
\mathrm{III} & \leq\left\|e_{u}^{0}\right\|_{H^{-1}(\Omega)}\left\|\int_{0}^{t^{m}} \nabla P_{h}^{1} e_{\theta}^{h}(t) d t\right\|_{L^{2}(\Omega)} \\
& \leq \frac{1}{4}\left\|\int_{0}^{t^{m}} \nabla P_{h}^{1} e_{\theta}^{h}(t) d t\right\|_{L^{2}(\Omega)}^{2}+\left\|e_{u}^{0}\right\|_{H^{-1}(\Omega)}^{2} .
\end{aligned}
$$

Term IV is essentially due to the extrapolation algorithm and requires some time regularity for temperature to be treated. Indeed, we exploit property $0 \leq \alpha_{\varepsilon}^{\prime} \leq \varepsilon^{-1}$ together with (4.1) and (2.12) to conclude

$$
\begin{aligned}
\mathrm{IV} & \leq C \frac{\tau}{\varepsilon} \sum_{n=1}^{m}\left\|\partial P_{h}^{0} \Theta^{n}\right\|_{L^{2}(\Omega)} \int_{I^{n}}\left(\left\|e_{\theta}^{h}(t)\right\|_{L^{2}(\Omega)}+\left\|\left[P_{h}^{1}-I\right] \theta_{\varepsilon}(t)\right\|_{L^{2}(\Omega)}\right) d t \\
& \leq \frac{\mu}{8}\left\|e_{\theta}^{h}\right\|_{L^{2}\left(0, t^{m} ; L^{2}(\Omega)\right)}^{2}+C \frac{\tau^{2}}{\varepsilon^{2}} \sum_{n=1}^{m} \tau\left\|\partial P_{h}^{0} \Theta^{n}\right\|_{L^{2}(\Omega)}^{2}+C h^{2}\left\|\theta_{\varepsilon}\right\|_{L^{2}\left(0, t^{m} ; H^{1}(\Omega)\right)} \\
& \leq \frac{\mu}{8}\left\|e_{\theta}^{h}\right\|_{L^{2}\left(0, t^{m} ; L^{2}(\Omega)\right)}^{2}+C\left(\frac{\tau^{2}}{\varepsilon^{2}}+h^{2}\right) .
\end{aligned}
$$

It only remains to examine the source term V. We make use again of (2.4) and (4.1) to bound $\mathrm{V}$ as follows:

$$
\begin{array}{r}
\mathrm{V} \leq \sum_{n=1}^{m}\left\{\sum_{i=1}^{n}\left(\int_{I^{i}}\left\|e_{\theta}^{h}(s)\right\|_{L^{2}(\Omega)} d s+\tau\left\|P_{h}^{0}\left[\Theta^{i}-\Theta^{i-1}\right]\right\|_{L^{2}(\Omega)}\right)\right. \\
\left.\times \int_{I^{n}}\left(\left\|e_{\theta}^{h}(t)\right\|_{L^{2}(\Omega)}+\left\|\left[P_{h}^{1}-I\right] \theta_{\varepsilon}(t)\right\|_{L^{2}(\Omega)}\right) d t\right\} \\
\leq \frac{\mu}{2}\left\|e_{\theta}^{h}\right\|_{L^{2}\left(0, t^{m} ; L^{2}(\Omega)\right)}^{2}+C \sum_{n=1}^{m} \tau\left\|e_{\theta}^{h}\right\|_{L^{2}\left(0, t^{n} ; L^{2}(\Omega)\right)}^{2}+C\left(h^{2}+\tau^{2}\right) .
\end{array}
$$

Inserting all the previous estimates in (5.4) results in the following inequality

$$
\begin{aligned}
& \mu\left\|e_{\theta}^{h}\right\|_{L^{2}\left(0, t^{m} ; L^{2}(\Omega)\right)}^{2}+\left\|\int_{0}^{t^{m}} P_{h}^{1} e_{\theta}^{h}(t) d t\right\|_{H_{0}^{1}(\Omega)}^{2} \\
& \leq C\left(\frac{h^{2}}{\varepsilon}+\frac{\tau^{2}}{\varepsilon^{2}}+\tau+\left\|e_{u}^{0}\right\|_{H^{-1}(\Omega)}^{2}\right)+C \sum_{n=1}^{m} \tau\left\|e_{\theta}^{h}\right\|_{L^{2}\left(0, t^{n} ; L^{2}(\Omega)\right)}^{2} .
\end{aligned}
$$

Finally, application of the discrete Gronwall lemma enables us to control the rightmost term. We further exploit the fact that $\int_{0}^{t} \theta_{\varepsilon}(s) d s \in L^{\infty}\left(0, T ; H^{2}(\Omega)\right)$ to get rid of $P_{h}^{1}$ on the left-hand side; this comes from integrating $(2.15)$ on $(0, t)$ and using $\left(\mathbf{H}_{\Omega}\right)$ coupled with (2.12). Moreover, since $\int_{0}^{t} \theta_{\varepsilon}(s) d s \in H^{1}\left(0, T ; H_{0}^{1}(\Omega)\right) \subset$ $C^{0,1 / 2}\left(0, T ; H_{0}^{1}(\Omega)\right)$, we readily obtain the desired estimate (5.2).

We now complete the error analysis with an error estimate for enthalpy.

THEOREM 5.3. There exists a constant $C>0$ independent of $h, \varepsilon$ and $\tau$ such that

$$
\left\|e_{u}^{h}\right\|_{L^{\infty}\left(0, T ; H^{-1}(\Omega)\right)} \leq C\left(h \varepsilon^{-1 / 2}+\tau \varepsilon^{-1}+\tau^{1 / 2}+\left\|e_{u}^{0}\right\|_{H^{-1}(\Omega)}\right)
$$


Proof. This result is a simple consequence of (5.2). To see why, write (5.3) as follows:

$$
\begin{aligned}
\left\langle u_{\varepsilon}^{n}-\right. & \left.U^{n}, \phi\right\rangle+\left\langle\nabla \int_{0}^{t^{n}} e_{\theta}^{h}(t) d t, \nabla \phi\right\rangle \\
& =\left\langle e_{u}^{0}, \phi\right\rangle+\left\langle\sum_{i=1}^{n} \int_{I^{i}}\left[f\left(\theta_{\varepsilon}(t)\right)-f\left(P_{h}^{0} \Theta^{i-1}\right)\right] d t, \phi\right\rangle, \quad \forall \phi \in \mathbf{V}_{h}^{1},
\end{aligned}
$$

where we have used definition (2.17) of $U^{n}$. Let $\phi \in H_{0}^{1}(\Omega)$ be given, and denote by $\phi_{h} \in \mathrm{V}_{h}^{1}$ a suitable interpolant of $\phi$ satisfying $\left\|\phi-\phi_{h}\right\|_{H^{s}(\Omega)} \leq C h^{1-s}\|\phi\|_{H^{1}(\Omega)}, s=$ $0,1[2]$. Then,

$$
\begin{aligned}
\left\langle u_{\varepsilon}^{n}-U^{n}, \phi\right\rangle= & \left\langle u_{\varepsilon}^{n}-U^{n}, \phi-\phi_{h}\right\rangle-\left\langle\nabla \int_{0}^{t^{n}} e_{\theta}^{h}(t) d t, \nabla \phi_{h}\right\rangle \\
& +\left\langle e_{u}^{0}, \phi_{h}\right\rangle+\left\langle\sum_{i=1}^{n} \int_{I^{i}}\left[f\left(\theta_{\varepsilon}(t)\right)-f\left(P_{h}^{0} \Theta^{i-1}\right)\right] d t, \phi_{h}\right\rangle \\
\leq & C\left(h \tau^{1 / 2} \varepsilon^{-1 / 2}+h \varepsilon^{-1 / 2}+\tau \varepsilon^{-1}+\tau^{1 / 2}+\left\|e_{u}^{0}\right\|_{H^{-1}(\Omega)}\right)\|\phi\|_{H_{0}^{1}(\Omega)},
\end{aligned}
$$

as results from (2.12), (4.6) and (5.2). This shows (5.5), because

$$
e_{u}^{h}(t)=u_{\varepsilon}^{n}-U^{n}+u_{\varepsilon}(t)-u_{\varepsilon}^{n}, \quad \forall t \in I^{n},
$$

and $H^{1}\left(0, T ; H^{-1}(\Omega)\right) \subset C^{0,1 / 2}\left(0, T ; H^{-1}(\Omega)\right)$, so we can apply $(2.12)$.

We conclude this section by establishing the global rate of convergence of the proposed extrapolation method.

COROLlaRY 5.1. Let $h, \varepsilon$ and $\tau$ satisfy $\varepsilon=C_{1} h$ and $\tau=C_{2} h^{3 / 2}$, where $C_{1}, C_{2}$ indicate arbitrary positive constants. If $\Theta^{0}$ is chosen as in Section 3 , then

$$
\left\|e_{\theta}\right\|_{L^{2}(Q)}+\left\|\int_{0}^{t} e_{\theta}\right\|_{L^{\infty}\left(0, T ; H_{0}^{1}(\Omega)\right)}+\left\|e_{u}\right\|_{L^{\infty}\left(0, T ; H^{-1}(\Omega)\right)}=O\left(h^{1 / 2}\right) .
$$

Remark 5.1. In view of (1.6) and (2.17) we realize that $\tau$ must tend to 0 faster than $\varepsilon$, therefore than $h$, for the discrete phase variable $\chi^{n}$ to converge to the correct limit. This, in conjunction with the fact that Problem $(\mathbf{P})$ is not purely parabolic, partially justifies the relation $\tau=C_{2} h^{3 / 2}$.

6. Incomplete Iteration Process. The object of this section is to show that whenever an iterative procedure is to be employed, Problem $\left(\mathbf{P}_{\varepsilon, h, \tau}\right)$ does not need to be solved exactly but rather approximately in the sense that only a prescribed error reduction must be imposed. This reduces considerably the computational labor and still preserves the overall accuracy discussed in Section 5. Our result is in the spirit of a former one in [6] for the standard extrapolation method, but the present proof is quite different from that in [6] because of the intrinsic lack of regularity associated with degenerate parabolic equations.

Suppose that the linear equation (2.16) is solved with an iterative algorithm, such as the conjugate gradient method, and that the initial guess is $\Theta^{n-1}$. Then 
the incomplete iteration reads as follows:

Problem $\left(\widetilde{\mathbf{P}}_{\varepsilon, h, \tau}\right)$. For $1 \leq n \leq N$ let $\Xi^{n} \in \mathbf{V}_{h}^{1}$ denote the exact solution to

$$
\begin{aligned}
& \left(\mu+\varepsilon^{-1}\right) \tau^{-1}\left\langle P_{h}^{0} \Xi^{n}-P_{h}^{0} \Theta^{n-1}, \phi\right\rangle+\left\langle\nabla \Xi^{n}, \nabla \phi\right\rangle \\
& \quad=\left\langle\partial \alpha_{\varepsilon}\left(P_{h}^{0} \Theta^{n-1}\right), \phi\right\rangle+\left\langle f\left(P_{h}^{0} \Theta^{n-1}\right), \phi\right\rangle, \quad \forall \phi \in \mathbf{V}_{h}^{1} .
\end{aligned}
$$

Then, define $\Theta^{n} \in \mathbf{V}_{h}^{1}$ to be any iteration satisfying

$$
\begin{aligned}
\| \Xi^{n}- & \Theta^{n} \|_{\mathbf{A}} \\
& :=\left\{\left(\mu+\varepsilon^{-1}\right) \tau^{-1}\left\|P_{h}^{0}\left[\Xi^{n}-\Theta^{n}\right]\right\|_{L^{2}(\Omega)}^{2}+\left\|\nabla\left[\Xi^{n}-\Theta^{n}\right]\right\|_{L^{2}(\Omega)}^{2}\right\}^{1 / 2} \\
& \leq 2 \tau\left\|\Xi^{n}-\Theta^{n-1}\right\|_{\mathbf{A}} .
\end{aligned}
$$

Inequality (6.2) is the typical error reduction produced by the conjugate gradient method and can be transformed as follows:

$$
\left\|\Xi^{n}-\Theta^{n}\right\|_{\mathbf{A}} \leq 4 \tau\left\|\Theta^{n}-\Theta^{n-1}\right\|_{\mathbf{A}} .
$$

This results from taking $\tau \leq 1 / 4$. The error reduction (6.2) is the basic ingredient for stability and accuracy to be maintained, as we shall prove below. Whenever the conjugate gradient method is utilized, (6.2) provides the lower bound (2.21) for the number of required iterations.

Equation (6.1) can also be expressed in the following equivalent form:

$$
\begin{aligned}
& \left(\mu+\varepsilon^{-1}\right)\left\langle\partial P_{h}^{0} \Theta^{n}, \phi\right\rangle+\left\langle\nabla \Theta^{n}, \nabla \phi\right\rangle \\
& \quad=\left\langle\partial \alpha_{\varepsilon}\left(P_{h}^{0} \Theta^{n-1}\right), \phi\right\rangle+\left\langle f\left(P_{h}^{0} \Theta^{n-1}\right), \phi\right\rangle+\left\langle R^{n}, \phi\right\rangle, \quad \forall \phi \in \mathbf{V}_{h}^{1},
\end{aligned}
$$

where $R^{n} \in H^{-1}(\Omega)$ is defined by

$$
\begin{aligned}
\left\langle R^{n}, \phi\right\rangle:= & \left(\mu+\varepsilon^{-1}\right) \tau^{-1}\left\langle P_{h}^{0}\left[\Theta^{n}-\Xi^{n}\right], \phi\right\rangle \\
& +\left\langle\nabla\left[\Theta^{n}-\Xi^{n}\right], \nabla \phi\right\rangle, \quad \forall \phi \in H_{0}^{1}(\Omega) .
\end{aligned}
$$

The only novelty in analyzing stability and accuracy is the presence of the remainder $R^{n}$; so most details will be skipped. The first result extends Lemma 4.1 to this more general situation.

LEMMA 6.1. Assume $\tau=o(\varepsilon)$. Then there exists a constant $C>0$ independent of $h, \varepsilon$ and $\tau$ such that

$$
\begin{aligned}
\sum_{n=1}^{N} \tau\left\|\partial P_{h}^{0} \Theta^{n}\right\|_{L^{2}(\Omega)}^{2} & +\sum_{n=1}^{N}\left\|\nabla\left[\Theta^{n}-\Theta^{n-1}\right]\right\|_{L^{2}(\Omega)}^{2} \\
& +\max _{1 \leq n \leq N}\left\|\nabla \Theta^{n}\right\|_{L^{2}(\Omega)}^{2} \leq C\left(1+\left\|\nabla \Theta^{0}\right\|_{L^{2}(\Omega)}^{2}\right) .
\end{aligned}
$$

Proof. Take $\phi:=\tau \partial \Theta^{n} \in \mathrm{V}_{h}^{1}$ as a test function in (6.4), add on $n$ from 1 to $m \leq N$ and recall definition (2.3) of $P_{h}^{0}$ as well as the notation in (4.2) to write the resulting expression as $\mathrm{I}+\mathrm{II}=\mathrm{III}+\mathrm{IV}+\mathrm{V}$, where

$$
\mathrm{V}=\left(\mu+\varepsilon^{-1}\right) \sum_{n=1}^{m}\left\langle P_{h}^{0}\left[\Theta^{n}-\Xi^{n}\right], \partial \Theta^{n}\right\rangle+\sum_{n=1}^{m}\left\langle\nabla\left[\Theta^{n}-\Xi^{n}\right], \nabla\left[\Theta^{n}-\Theta^{n-1}\right]\right\rangle .
$$

The analysis of the first four terms is similar to that in Lemma 4.1. In particular, we now need the full expression of II, namely,

$$
\mathrm{II}=\frac{1}{2}\left\|\nabla \Theta^{m}\right\|_{L^{2}(\Omega)}^{2}-\frac{1}{2}\left\|\nabla \Theta^{0}\right\|_{L^{2}(\Omega)}^{2}+\frac{1}{2} \sum_{n=1}^{m}\left\|\nabla\left[\Theta^{n}-\Theta^{n-1}\right]\right\|_{L^{2}(\Omega)}^{2} .
$$


We next evaluate the contribution due to V. By virtue of (6.3), we have

$$
\begin{aligned}
\mathrm{V} \leq & \frac{\mu}{4} \sum_{n=1}^{m} \tau\left\|\partial P_{h}^{0} \Theta^{n}\right\|_{L^{2}(\Omega)}^{2}+\frac{1}{4} \sum_{n=1}^{m}\left\|\nabla\left[\Theta^{n}-\Theta^{n-1}\right]\right\|_{L^{2}(\Omega)}^{2} \\
& +\left(\mu+\varepsilon^{-1}\right)^{2} \mu^{-1} \tau^{-1} \sum_{n=1}^{m}\left\|P_{h}^{0}\left[\Theta^{n}-\Xi^{n}\right]\right\|_{L^{2}(\Omega)}^{2}+\sum_{n=1}^{m}\left\|\nabla\left[\Theta^{n}-\Xi^{n}\right]\right\|_{L^{2}(\Omega)}^{2} \\
\leq & \left(\frac{\mu}{4}+C \frac{\tau^{2}}{\varepsilon^{2}}\right) \sum_{n=1}^{m} \tau\left\|\partial P_{h}^{0} \Theta^{n}\right\|_{L^{2}(\Omega)}^{2}+\left(\frac{1}{4}+C \frac{\tau^{2}}{\varepsilon}\right) \sum_{n=1}^{m}\left\|\nabla\left[\Theta^{n}-\Theta^{n-1}\right]\right\|_{L^{2}(\Omega)}^{2}
\end{aligned}
$$

Collecting the above estimates and exploiting the fact that $\tau=o(\varepsilon)$, we easily derive the assertion after applying the discrete Gronwall lemma.

We are now in a position to prove energy error estimates which generalize those in Corollary 5.1.

THEOREM 6.1. Let $h, \varepsilon, \tau$ and $\Theta^{0}$ be chosen as in Corollary 5.1. Then

$$
\left\|e_{\theta}\right\|_{L^{2}(Q)}+\left\|\int_{0}^{t} e_{\theta}\right\|_{L^{\infty}\left(0, T ; H_{0}^{1}(\Omega)\right)}+\left\|e_{u}\right\|_{L^{\infty}\left(0, T ; H^{-1}(\Omega)\right)}=O\left(h^{1 / 2}\right) .
$$

Proof. We proceed along the same lines as those in Theorems 5.2 and 5.3. In particular, instead of (5.4) we now have the equality $\mathrm{I}+\mathrm{II}=\mathrm{III}+\mathrm{IV}+\mathrm{V}+\mathrm{VI}$, where VI stands for

$$
\begin{aligned}
\mathrm{VI}: & =\sum_{n=1}^{m} \tau\left\langle\sum_{i=1}^{n} R^{i}, \int_{I^{n}} P_{h}^{1} e_{\theta}^{h}(t) d t\right\rangle \\
= & \sum_{n=1}^{m}\left(\mu+\varepsilon^{-1}\right)\left\langle\sum_{i=1}^{n} P_{h}^{0}\left[\Theta^{i}-\Xi^{i}\right], \int_{I^{n}} P_{h}^{1} e_{\theta}^{h}(t) d t\right\rangle \\
& +\sum_{n=1}^{m} \tau\left\langle\sum_{i=1}^{n} \nabla\left[\Theta^{i}-\Xi^{i}\right], \nabla \int_{I^{n}} P_{h}^{1} e_{\theta}^{h}(t) d t\right\rangle .
\end{aligned}
$$

Terms I to $\mathrm{V}$ can be bounded in the same manner as in Theorem 5.2, with II verifying

$$
\mathrm{II}=\frac{1}{2}\left\|\int_{0}^{t^{m}} \nabla P_{h}^{1} e_{\theta}^{h}(t) d t\right\|_{L^{2}(\Omega)}^{2}+\frac{1}{2} \sum_{n=1}^{m}\left\|\nabla \int_{I^{n}} P_{h}^{1} e_{\theta}^{h}(t) d t\right\|_{L^{2}(\Omega)}^{2} .
$$

Next, we make use of (2.4), in conjunction with (2.12) and (6.6), to split term VI as follows:

$$
\begin{aligned}
\mathrm{VI} \leq & \delta\left\|e_{\theta}^{h}\right\|_{L^{2}\left(0, t^{m} ; L^{2}(\Omega)\right)}^{2}+\delta \sum_{n=1}^{m}\left\|\nabla \int_{I^{n}} P_{h}^{1} e_{\theta}^{h}(t) d t\right\|_{L^{2}(\Omega)}^{2}+C \delta h^{2} \\
& +C \delta^{-1}\left(\mu+\varepsilon^{-1}\right)^{2} \sum_{n=1}^{m} \sum_{i=1}^{n}\left\|P_{h}^{0}\left[\Theta^{i}-\Xi^{i}\right]\right\|_{L^{2}(\Omega)}^{2} \\
& +C \delta^{-1} \tau \sum_{n=1}^{m} \sum_{i=1}^{n}\left\|\nabla\left[\Theta^{i}-\Xi^{i}\right]\right\|_{L^{2}(\Omega)}^{2},
\end{aligned}
$$

where $\delta>0$ is to be selected. The last two terms can be further bounded by

$$
C \delta^{-1} \frac{\tau^{2}}{\varepsilon^{2}} \sum_{n=1}^{m} \tau \sum_{i=1}^{n}\left(\tau\left\|\partial P_{h}^{0} \Theta^{i}\right\|_{L^{2}(\Omega)}^{2}+\left\|\nabla\left[\Theta^{i}-\Theta^{i-1}\right]\right\|_{L^{2}(\Omega)}^{2}\right) \leq C \delta^{-1} \frac{\tau^{2}}{\varepsilon^{2}},
$$


as results from (6.3) and (6.6). A proper choice of $\delta$ allows the corresponding terms to be absorbed into the left-hand side of (5.4), thus yielding the first two estimates in (6.7). The remaining one can be obtained by coupling the argument in Theorem 5.3 with that above to handle $\sum_{i=1}^{n} \tau R^{i}$.

Department of Mathematics and

Institute for Physical Science and Technology

University of Maryland

College Park, Maryland 20742

E-mail: rhn\%julia.umd.edu@eneevax.umd.edu

1. O. Axelsson \& V. A. Barker, Finite Element Solution of Boundary Value Problems: Theory and Applications, Academic Press, Orlando, FL, 1984.

2. P.G. Ciarlet, The Finite Element Method for Elliptic Problems, North-Holland, Amsterdam, 1978.

3. J. Douglas, JR. \& T. Dupont, "Galerkin methods for parabolic equations," SIAM J. Numer. Anal., v. 7, 1970, pp. 575-626.

4. J. DouglaS, JR. \& T. DUPONT, "Alternating-direction Galerkin methods on rectangles," in Numerical Solutions of Partial Differential Equations, vol. II (B. Hubbard, ed.), Academic Press, New York, 1971, pp. 133-214.

5. J. Douglas, JR. \& T. DuponT, "Galerkin methods for parabolic equations with nonlinear boundary conditions," Numer. Math., v. 20, 1973, pp. 213-217.

6. J. DOUGLAS, JR., T. DUPONT \& R. EWING, "Incomplete iteration for time-stepping a Galerkin method for a quasilinear parabolic problem," SIAM J. Numer. Anal., v. 16, 1979, pp. 503-522.

7. A. Friedman, "The Stefan problem in several space variables," Trans. Amer. Math. Soc., v. 133,1968, pp. $51-87$.

8. J. W. JEROME \& M. ROSE, "Error estimates for the multidimensional two-phase Stefan problem," Math. Comp., v. 39, 1982, pp. 377-414.

9. M. LUSKIN, "A Galerkin method for nonlinear parabolic equations with nonlinear boundary conditions," SIAM J. Numer. Anal., v. 16, 1979, pp. 284-299.

10. E. Magenes, "Problemi di Stefan bifase in più variabili spaziali," Matematiche, v. 36, 1981, pp. 65-108.

11. E. MAGENES, "Remarques sur l'approximation des problèmes non linéaires paraboliques," in Analyse Mathématique et Applications (volume dedicated to J.L.Lions), Gauthier-Villars, Paris, 1988, pp. 297-318.

12. E. Magenes, R. H. NochetTo \& C. Verdi, "Energy error estimates for a linear scheme to approximate nonlinear parabolic problems," RAIRO Modél. Math. Anal. Numér., v. 21, 1987, pp. $655-678$.

13. R. H. Nochetto, "Error estimates for two-phase Stefan problems in several space variables, I: Linear boundary conditions," Calcolo, v. 22, 1985, pp. 457-499.

14. R. H. Noсhетto, "Error estimates for multidimensional singular parabolic problems," Japan. J. Appl. Math., v. 4, 1987, pp. 111-138.

15. R. H. NochetTo, "Numerical methods for free boundary problems," in Free Boundary Problems: Theory and Applications (K. H. Hoffmann and J. Sprekels, eds.), vols. V, VI, Research Notes in Math., Longman, London, 1988. (To appear.)

16. R. H. NOCHETTO \& C. VERDI, "An efficient linear scheme to approximate parabolic free boundary problems: Error estimates and implementation," Math. Comp., v. 51, 1988, pp. 27-53.

17. R. H. NOChetTo \& C. Verdi, "The combined use of a nonlinear Chernoff formula with a regularization procedure for two-phase Stefan problems," Numer. Funct. Anal. Optim., v. 9, 1987-88, pp. 1177-1192.

18. M. PAOLINI, G. SACCHI \& C. VERDI, "Finite element approximations of singular parabolic problems," Internat. J. Numer. Methods Engrg., v. 26, 1988, pp. 1989-2007.

19. M. Rose, "Numerical methods for flows through porous media, I," Math. Comp., v. 40, 1983, pp. 435-467. 